\title{
Research on Key Problems of Heuristic Evolutionary Algorithm for Dynamic High Dimensional Multi-Objective Optimization Problem Yang Li and Liping Wang
}

\author{
Pingxiang University, Pingxiang, Jiangxi 337000
}

\begin{abstract}
Keywords: Multi-objective optimization; Evolutionary algorithm; Target reduction algorithm; Dynamic high dimension
\end{abstract}

\begin{abstract}
The multi-objective optimization method has a strong background of engineering application. In recent years, the problem of solving multi-objective optimization based on evolutionary algorithm has become a hot topic in the international academic circle. This paper studies the problem of dynamic application of high-dimensional multi-objective optimization problem heuristic evolutionary algorithm key technology and algorithm, which constructs online target dimension reduction algorithm model, alternating between the executive, the original problem is gradually transformed into smaller problems, improve the optimization algorithm, which provides a new method for solving high dimension the multi-objective optimization problem.
\end{abstract}

\section{Introduction}

Multi-objective optimization is one of the most common problems in engineering practice and daily life. It has been widely concerned by the academia and the engineering community. Compared with single objective optimization problem, only one objective function is considered [1]. Multi-objective optimization problems need to optimize multiple objective functions simultaneously, and these objectives usually exist independently, but restrict each other and restrict each other [2, 3]. In other words, the performance of other targets will be reduced while some target is improved, which makes it more difficult to solve this kind of algorithm. Initially, for multi-objective optimization problems, most of them adopted some integration methods to transform multi-objective optimization problems into single objective optimization problems $[4,5]$. Then, a more mature single objective optimization algorithm was applied to solve them.

\section{Fitness Evaluation and Selection Strategy in Multi-Objective Optimization Evolutionary Algorithm}

Fitness evaluation and selection strategy is one of the key technologies of multi-objective optimization evolutionary algorithm. It plays a very important role in avoiding individual gene loss and improving the search speed and convergence performance of algorithm. The most commonly used solution is to sort the population of evolutionary algorithm based on the concept of Pareto dominance, calculate the fitness of each individual by ranking values, and select operation according to the fitness of individuals [6]. Because Pareto dominance concept has all the same importance as all objectives without introducing any preference information, and it is a strong sorting relation, the algorithm often produces a lot of non-dominated solutions that can not be compared to the best degree after operation [7]. Many non-dominated solutions can not be directly applied in practical engineering design, and decision-makers need to choose a satisfactory solution as the final solution of the problem, which undoubtedly increases the decision burden of decision makers. In addition, the concept of Pareto dominance ranking method to effectively deal with high dimensional optimization problems a number of greater than or equal to the multi-objective evolutionary algorithm based on the multiple non dominated solutions in finite size groups, these non-dominated solutions determined by lack of comparative standards, to the selection of operation in leading the algorithm stagnation [8].

Evolutionary algorithm is a kind of random search algorithm that draws on biological selection and natural genetic mechanism. The proportion of non-dominant solutions in the random initial population ( $\mathrm{N}$ representation of population size) is shown in Figure 1. It has two important 
characteristics: group search strategy and information exchange among individuals. Multi objective evolutionary algorithm compared with the traditional method, its advantage lies in: in the implementation of a algorithm to obtain a set of optimal solutions, to avoid most of the traditional methods need to run multiple times and bring huge time overhead; the algorithm is not affected by the application of Pareto optimal front shape; evolutionary algorithm used to evaluate the objective function of the individual advantages and disadvantages of different problem solving, you only need to design the corresponding fitness function, without modifying the other part of the algorithm has good universality $[9,10]$. At present, many efficient multi-objective evolutionary algorithms have been widely used in engineering practice, production and life.

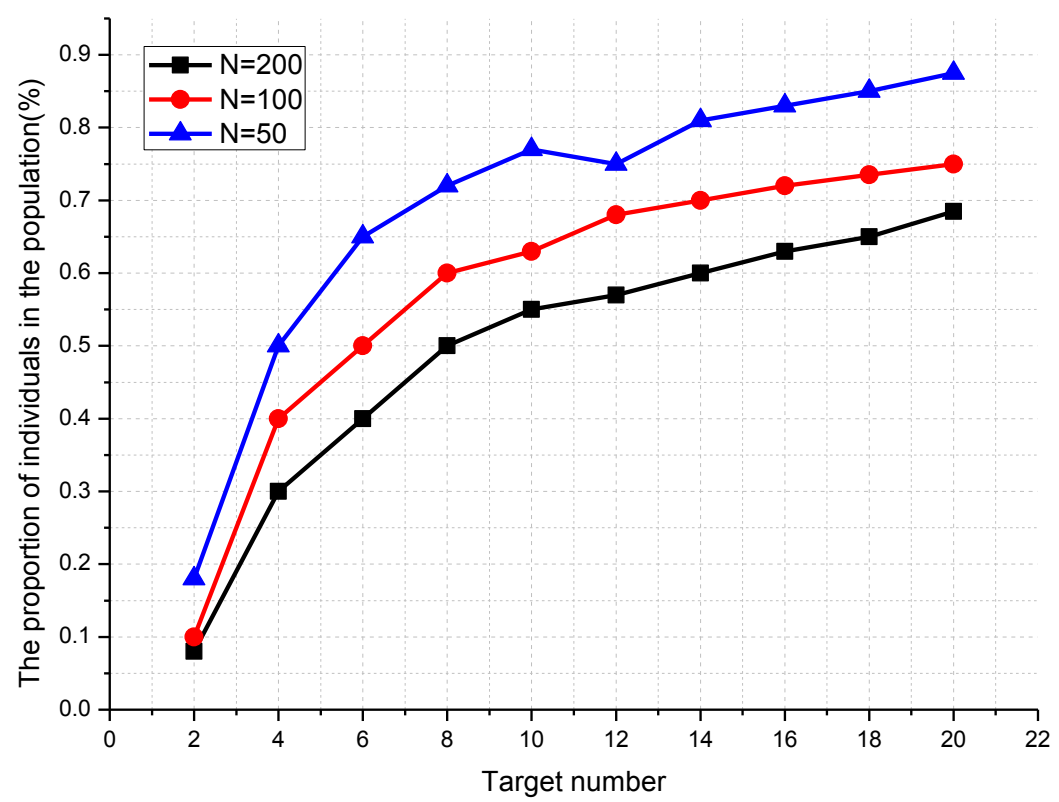

Figure 1. The proportion of non-dominant solutions in the random generated initial population (N represents the population size)

Fuzzy system is based on natural language and expert experience. It allows the use of imprecise data. Its expression and reasoning process are very similar to human thinking characteristics. Therefore, fuzzy system can deal with problems quickly and conveniently, and is easy to understand conceptually. Because of the complexity of objective things and the fuzziness of human thinking, in general, people can only provide qualitative language descriptions for preference information among different targets. Meanwhile, there is a subjective bias in the mutual improvement of the two solutions. Therefore, by introducing fuzzy information into the multi-objective optimization evolutionary algorithm, we can introduce preference information and improve the amplitude to compare the solution, so as to overcome the limitation of the concept, it is an appropriate choice [11].

For a given optimization problem, the basic steps to solve the problem by using the multi-objective optimization evolutionary algorithm are as follows

(1) The multi-objective optimization model of the problem is extracted. According to the actual requirements of the problem, determine the number of target problems to be optimized if possible, mathematical expression is given the optimization goal, and through mathematical transform such as the reciprocal, the optimization object all were converted to the maximization or minimization problem.

(2) Improved algorithm. Aiming at the characteristics of a given problem, the local modifications based on the prior knowledge of the problem to the multi-objective optimization evolutionary algorithm are mainly individual coding, initialization, initialization, crossover, mutation and constraint handling.

(3) The multi-objective optimization problem is solved. The parameters of multi-objective optimization evolutionary algorithm are determined. In certain software environment, programming is implemented to improve the algorithm for the problem characteristics; given 
the input parameters of the algorithm, operation algorithm, and output the running results. In the operation of the algorithm, the target value of the different individuals in the group needs to be evaluated $[12,13]$.

(4) Analysis results. The output data of the algorithm is sorted out, and the performance of the algorithm is analysed through performance measurement and comparison with existing algorithms, which verifies the effectiveness of the algorithm in a given optimization problem.

\section{High Dimensional Multi-Objective Optimization Problem Solving Method Based on Online Objective Reduction}

(1) On-line target dimensionality reduction algorithm model

When solving many high-dimensional multi-objective optimization problems in practical applications, it not only requires the multi-objective optimization algorithm to have good convergence and distribution, but also needs to consider two important factors. One is because of cost considerations, for computing resources and computer performance have certain limitations, which requires calculation of multi-objective optimization algorithm with low complexity, small amount of calculation; the two is some application problems to have higher real-time requirements, which requires the multi-objective optimization algorithm can in a relatively short period of time are satisfactory the algorithm is also requires a shorter running time or faster convergence speed [14]. The increase of target number not only makes it difficult to obtain satisfactory results for high dimensional multi-objective optimization problems, but also has high computational complexity and long running time. If the problem can be converted to a small target, it is bound to improve the effectiveness and efficiency of the solution.

As a result, a new method of solving high dimensional multi-objective optimization problem is proposed, which is called the online target reduction algorithm. The algorithm divides the object dimension reduction algorithm is introduced to the search process of multi-objective evolutionary algorithm, the non-dominated solution set goal of dimensionality reduction algorithm to obtain the target for a subset of the original problem, the multi-objective optimization algorithm only in the reduced target set search optimal solution, gradually reduces the size of the problem until unable to delete [15]. The online target reduction algorithm model is shown in Figure 2.

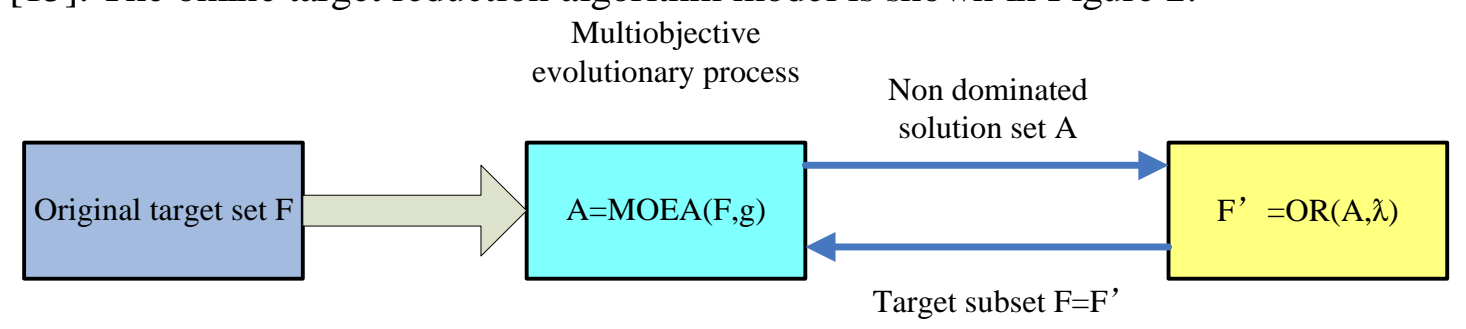

Figure 2. On line target reduction algorithm model

Assuming that the current target set is $F$, the multi objective evolutionary algorithm evolves the $g$ generation on the $F$ and obtains a set of non-dominated solutions set $A$, namely:

$$
A=\operatorname{MOEA}(F, g)
$$

MOEA represents a multi-objective evolutionary algorithm.

The target dimensionality reduction algorithm is based on the non-dominated solution set obtained by multi-objective evolutionary algorithm as input sample data. Based on $F$, we find a subset of $F$ target consisting of fewer targets.

$$
\begin{gathered}
F^{\prime}=O R(A, \lambda) \\
F=F^{\prime}
\end{gathered}
$$

OR represents the target reduction algorithm, and $\lambda$ is the parameter of the target reduction algorithm, which is given by the decision-maker in advance. When a given number of target $\lambda$ 
reduced, OR algorithm is used to find composed of $|F|-\lambda$ target minimum error target subset. When $\lambda$ is given as the original problem to change the error threshold, the OR algorithm is used to find the smallest subset of the target which is not more than $\lambda$.

\section{Summary}

The high dimensional multi-objective optimization problem with more than 3 targets is a common problem form in the field of practical engineering and scientific research. Such problems usually have many conflicting goals, which are highly demanding for computer performance, computing resources (such as storage devices) and real-time. Therefore, high-dimensional multi-objective evolutionary algorithm with strong search ability and high efficiency is of great application value. The research work of this paper is mainly focused on the key issues of heuristic evolutionary algorithm for dynamic high-dimensional multi-objective optimization problems. The online target dimension reduction method is mainly used in this paper.

\section{Acknowledgements}

This work was supported by Pingxiang Science and Technology Plan Project（2017GY004）,and 2017 Key Projects for R\&D in Jiangxi Province (20171BBE50049).

\section{References}

[1] Zavala G R, Nebro A J, Luna F, et al. A survey of multi-objective metaheuristics applied to structural optimization[J]. Structural \& Multidisciplinary Optimization, 2013, 49(4):537-558.

[2] Chevrier R, Liefooghe A, Jourdan L, et al. Solving a dial-a-ride problem with a hybrid evolutionary multi-objective approach: Application to demand responsive transport[J]. Applied Soft Computing, 2012, 12(4):1247-1258.

[3] Wang W, Feng X. Research on Internal Layout Optimization of Logistics Node under the Conditions of Complex Terrain Based on Computer Vision and Geographical Simulation System[J]. Abstract \& Applied Analysis, 2014, 2012(3):1395-1416.

[4] Shahab R. Comparative analysis of multiobjective evolutionary algorithms for random and correlated instances of multiobjective d-dimensional knapsack problems[J]. European Journal of Operational Research, 2011, 211(3):466-479.

[5] Sabar N R, Ayob M, Kendall G, et al. A Dynamic Multiarmed Bandit-Gene Expression Programming Hyper-Heuristic for Combinatorial Optimization Problems.[J]. IEEE Trans Cybern, 2015, 45(2):217-228.

[6] Bakhtiari H, Karimi M, Rezazadeh S. Modeling, analysis and multi-objective optimization of twist extrusion process using predictive models and meta-heuristic approaches, based on finite element results[J]. Journal of Intelligent Manufacturing, 2016, 27(2):1-11.

[7] Özkiş A, Babalik A. A novel metaheuristic for multi-objective optimization problems: The Multi-Objective Vortex Search algorithm[J]. Information Sciences, 2017, 402:124-148.

[8] Zk A, Babalk A. A novel metaheuristic for multi-objective optimization problems[J]. Information Sciences, 2017, 402:124-148.

[9] Mirjalili S. Dragonfly algorithm: a new meta-heuristic optimization technique for solving single-objective, discrete, and multi-objective problems[J]. Neural Computing \& Applications, 2016, 27(4):1053-1073.

[10] Mirjalili S, Jangir P, Saremi S. Multi-objective ant lion optimizer: a multi-objective optimization algorithm for solving engineering problems[J]. Applied Intelligence, 2016:1-17.

[11] Mehdizadeh E, Niaki S V D, Rahimi V. A vibration damping optimization algorithm for solving a new multi-objective dynamic cell formation problem with workers training[J]. Computers \& Industrial Engineering, 2016, 101. 
[12] Suksonghong K, Boonlong K. Multi-objective Cooperative Coevolutionary Algorithm with Dynamic Species-Size Strategy[J]. 2018:3-17.

[13] Camillo M H M, Romero M E V, Fanucchi R Z, et al. A Multi-objective Evolutionary Algorithm with Efficient Data Structure and Heuristic Initialization for Fault Service Restoration[J]. Procedia Computer Science, 2016, 80:2367-2371.

[14] Mirjalili S, Saremi S, Mirjalili S M, et al. Multi-objective grey wolf optimizer: A novel algorithm for multi-criterion optimization[J]. Expert Systems with Applications, 2016, 47:106-119.

[15] Singh M R, Singh M, Mahapatra S S, et al. Particle swarm optimization algorithm embedded with maximum deviation theory for solving multi-objective flexible job shop scheduling problem[J]. International Journal of Advanced Manufacturing Technology, 2016, 85(9-12):2353-2366. 